\title{
Device-free Multiple People Localization through Floor Vibration
}

\author{
Laixi Shi \\ laixis@andrew.cmu.edu \\ Carnegie Mellon University \\ Pittsburgh, PA
}

mostafa.mirshekari@gmail.comjfagert@andrew.cmu.edu

Carnegie Mellon University Carnegie Mellon University
Pittsburgh, PA

\author{
Mostafa Mirshekari \\ Pittsburgh, PA \\ Pei Zhang \\ peizhang@cmu.edu \\ Carnegie Mellon University \\ Pittsburgh, PA \\ Hae Young Noh \\ Carnegie Mellon University \\ Pittsburgh, PA
}

\author{
Yuejie Chi \\ yuejiechi@cmu.edu \\ Carnegie Mellon University \\ Pittsburgh, PA
}

\author{
Shijia Pan \\ span24@ucmerced.edu \\ University of California \\ Merced \\ Merced, CA
}

\begin{abstract}
Structural vibration-based human sensing provides an alternative approach for device-free human monitoring, which is used for healthcare, space and energy usage management, etc. Prior work on this approach mainly focused on one person walking scenarios, which limits their widespread application. The challenge with multiple walkers is that the observed vibration response is a mixture of each walker's footstep-induced response, and it is difficult to identify 1) how many concurrent walkers are present, and 2) the timing of their footstep impacts on the floor. As a result, the extraction of detailed location information for each walker is erroneous. To address this challenge, we propose a structure-informed vibration signal characterization method to enable the detection and localization of overlapping vibration signals induced by multiple concurrent walkers. The intuition is that, due to the randomness in people's behavior, their footsteps do not impact the floor exactly at the same time and overlap partially. We decompose the signal to a non-fundamental frequency band which contains the heel strike onset information. With this decomposed signal, we can identify the number of walkers and use the initial peak information to localize each person independently. We conducted real-world experiments with up to three concurrent walkers and our system achieved a detection rate of up to $90 \%$ and an average localization error of $0.65 \mathrm{~m}$ (2.9X baseline improvement).
\end{abstract}

\section{CCS CONCEPTS}

- Human-centered computing $\rightarrow$ Ubiquitous and mobile computing.

\section{KEYWORDS}

Ambient vibration sensing, multiple pedestrian localization, indoor localization, overlapping signal, TDOA

Permission to make digital or hard copies of all or part of this work for personal or classroom use is granted without fee provided that copies are not made or distributed for profit or commercial advantage and that copies bear this notice and the full citation on the first page. Copyrights for components of this work owned by others than ACM must be honored. Abstracting with credit is permitted. To copy otherwise, or republish, to post on servers or to redistribute to lists, requires prior specific permission and/or a fee. Request permissions from permissions@acm.org.

DFHS'19, November 10, 2019, New York, NY, USA

(c) 2019 Association for Computing Machinery.

ACM ISBN 978-1-4503-7007-3/19/11 . \$ \$15.00

https://doi.org/10.1145/3360773.3360887
ACM Reference Format:

Laixi Shi, Mostafa Mirshekari, Jonathon Fagert, Yuejie Chi, Hae Young Noh, Pei Zhang, and Shijia Pan. 2019. Device-free Multiple People Localization through Floor Vibration. In The 1st ACM International Workshop on DeviceFree Human Sensing (DFHS'19), November 10, 2019, New York, NY, USA. ACM, New York, NY, USA, 5 pages. https://doi.org/10.1145/3360773.3360887

\section{INTRODUCTION}

Indoor occupants localization enables various smart building applications such as building energy management and elderly/child monitoring. Various approaches have been explored for device-free localization, including vision $[1,3,10]$, pressure [22], infrared $[8,14]$, and radio $[2,21]$. These methods often rely on dense deployment or particular sensing requirements (e.g., line-of-sight). Researchers have been explored an alternative sensing modality through footstepinduced floor vibrations $[5,12,13,16,18,19]$. They demonstrate sub-meter level localization when a single person walks through the sensing area, or when at most two people walks in a small scope without considering the situation vibration signals overlap severely [16]. However, these works do not handle the situation when multiple people walk simultaneously and their footstep-induced floor vibration signals overlap and are difficult to detect and distinguish. Furthermore, the physical characteristics (e.g., structural natural frequency) of the propagation medium (i.e., the floor) make the structural vibration signal structure-dependent [7], thus making separating overlapping signals by traditional methods such as blind source separation (BSS) inapplicable [11]. Since we are focusing on the detection and localization with overlapping signals instead of localization refinement through sequential information, we will not discuss this aspect owing to space limitation.

In this paper, we present a system to enable multiple people localization using the following intuition: 1) the randomness of people's behavior will make their footstep-induced vibrations partially overlap even when they walk simultaneously, and 2) the footstepinduced signal onset shows high SNR and high damping rates in non-fundamental frequency bands (target scale band), which minimizes interference between multiple people's signals. Within this target scale band, our system conducts multiple footstep-induced signal onset detection and applies multi-dimensional scaling (MDS) on the extracted onsets to estimate footstep locations by minimizing a loss function. The major contributions of this work include: 

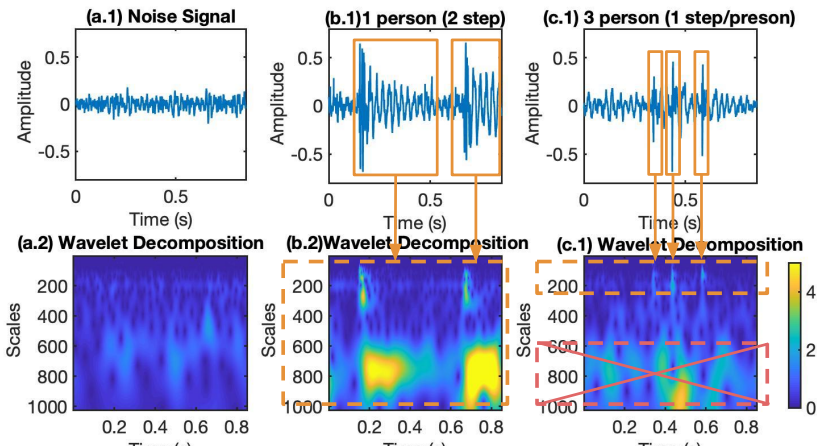

Figure 1: The first row shows the raw signal of ambient structural vibration signal (a.1), one person walking footstep-induced vibration signal (b.1) and three persons walking footstep-induced vibration signals that are partially overlapping (c.1). The second row shows their corresponding wavelet transforms.In (c.2), scales between 50 and 125 demonstrate three distinguishable footstep signal onsets, while the scales beyond 600 lose such time resolution.

- We present a device-free system to localize multiple people through floor vibrations.

- We utilize the intuition and domain knowledge on human behavior and structural response properties to detect the onsets of the partially overlapping footstep signals for localization.

- We evaluate the system through a series of real-world experiments and compare to baseline methods.

\section{PHYSICAL KNOWLEDGE}

We discuss the two intuitions - human behavior randomness and onset signal response in non-fundamental frequency band.

\subsection{Human Behavior Analysis}

The randomness of human behavior dictates that people walking in the same area are usually do not walking in perfect synchrony, (i.e., unlike military marching). As a result, their footstep-induced vibration signals may be partially overlapping instead of completely overlapping all the time [17]. Therefore, extraction signal onsets of partially overlapping signal is usually applicable.

In addition to the inference above, we also note that different people walk at different stepping frequencies [15] and with varying walking patterns [9]. Therefore, we infer that the heel strike timing for multiple pedestrians may not be completely overlapping, which enables us to identify a signal onset corresponding to each individual walker in the sensing area.

\subsection{Structural Response Characteristics}

To achieve multiple people detection and localization in overlapping vibration signals, we require an appropriate spectral subspace (target scale band) which provides high time resolution and SNR. Thus, the spectral properties of the instrumented floor are in demand.
To characterize the structural properties of the instrumented floor and identify an appropriate spectral subspace, we use a variation of a common structural identification approach, known as the Basic Frequency Domain (BFD) or "peak-picking" technique [4]. In our work, we use the continuous wavelet transform (CWT) to decompose the spectral components of the vibration responses in place of the traditional Fourier transform or Power Spectral Density estimation due to the fact that wavelet decomposition provides high time resolution in addition to frequency resolution. This high time-frequency resolution is advantageous over traditional frequency-only approaches because footstep-induced responses are time-varying (i.e., their spectral content may vary with time).

We placed a sensing node on the floor to record the ambient structural vibrations as well as the footstep-induced structural vibrations and compare their time and frequency components in Figure 1. Figure 1 shows the raw signal for structure vibrations in different scenarios and their corresponding wavelet decomposition. The wavelet decomposition is illustrated in the second row of Figure 1 , where the $\mathrm{x}$-axis is time, the $\mathrm{y}$-axis is the wavelet scale, and the colors represent the wavelet coefficient magnitude.

We observe in Figure 1 (b.2) that the footstep induced vibration signals have a concentration of energy between scales 700 to 1000 with a duration of $0.2 \mathrm{~s}$ and another energy concentration with a significantly shorter duration between scales 200 and 400 . These two spectral bands of energy concentration correspond to natural frequencies of the structure. Due to the concentrated energy and high SNR of the signals on the natural frequencies, prior work on human sensing utilized it for one person walking footstep detection.

However, when there are multiple impulsive signals overlapping, such selection criteria is inapplicable. Since the damped free vibration of natural frequency (usually $<30 \mathrm{~Hz}$ ) decays slowly, multiple peoples' signals at these frequencies have a high probability of overlapping. For instance, the three impulses from (c.1) and (c.2) overlap significantly on the scales corresponding to the structural natural frequency (i.e., beyond 600), making the detection on that scale difficult, if not impossible. Therefore, we select the scale range between 50 and 125 as our target scale band through the observation. For the three persons walking signal shown in Figure 1 (c.1) and (c.2), the three footstep-induced signal onsets have distinguishable time resolution between scales 50 and 125, as marked out with the orange dash-line box in Figure 1 (c.2).

\section{SYSTEM ARCHITECTURE}

Our structural vibration localization system consists of three modules as shown in Figure 2: vibration sensing, footstep event detection and onsets extraction, and multiple pedestrian step-level localization. In this section we describe each module in detail.

Structural Vibration Sensing: The vibration sensing module obtains floor vibration through multiple vibration sensing nodes placed at different locations. The system mainly consists of three parts: 1) a geophone sensor (which converts the surface vibration velocity into voltage signals); 2) an amplification board (to amplify the sensor output voltage signal so that the digitized signals will have sufficient resolution for analysis); and 3) analog-to-digital converter $(\mathrm{ADC})$ and data collection module. 


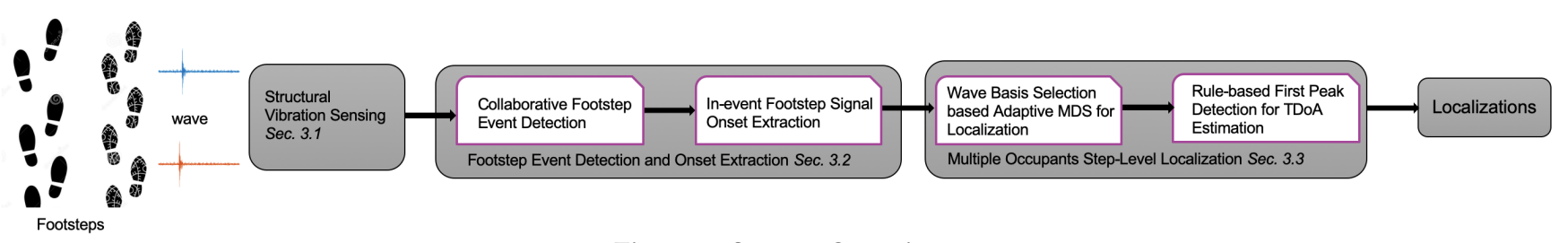

Figure 2: System Overview.

Footstep Event Detection and Onset Extraction: To localize multiple people's footstep through structural vibrations, our system first detects footstep events with the raw signal from a group of sensors. Then within a detected event, our system applies the CWT using Morlet wavelet as the mother wavelet. The selected wavelet has high waveform similarity with our target signal [12], which reduces dispersion effects and signal distortion. Lastly, with the target scale band, our system extracts footstep signal onsets from the partially overlapping signals and transmits the onset information to the localization module.

Multiple Occupant Step-Level Localization: Once the footstep onsets within a detected event are extracted, our system utilizes the wavelet decomposition of the extracted onsets signal for TDoA estimation. We conduct a rule-based peak detection to detect the first peak of the onset signals and estimate the TDoA between all pairwise sensors. Then, we utilize the TDoA values for the selected scales (target scale band on a range of wavelet scales) to further conduct adaptive multi-dimensional scaling (MDS) $[6,20]$ on these scales to estimate the localization of the footsteps.

\section{EXPERIMENTAL EVALUATION}

To evaluate our system, we conducted experiments in a school building hallway as shown in Figure 3.

Experimental Settings: We deployed our system with a group of four sensing nodes in a hallway to conduct experiments for evaluating our system. The experimental structure is a concrete slab on grade with a first observed natural frequency of $23.83 \mathrm{~Hz}$. Figure 3 shows the sensor deployment. Light blue circles indicate the sensor locations and the solid dash lines are the trajectories that pedestrian will step on during the real-world experiments. The target sensing area is approximately $3 \times 4 m$, where a pedestrian usually crosses through with six to seven steps. We use a camera placed in the hallway as the ground truth and marked the step location to guide occupants.

Metrics for System Performance: The evaluation metrics to measure the system performance are twofold: localization rate and localization accuracy. We calculate the Euclidean distance between the detected locations and the corresponding ground truth locations to measure the localization accuracy. When multiple footsteps occur simultaneously and the system fails to detect all of them, we compare the estimated locations to their closest ground truth location and calculate the error. We further evaluate the localization performance with the localization rate calculated as:

$$
\text { Localization_Rate }=\frac{\text { \#onsets localized in the sensing area }}{\text { \#onsets detected }}
$$

Pedestrian Walking Scenarios: We designed different pedestrian walking scenarios to demonstrate the robustness of the system.

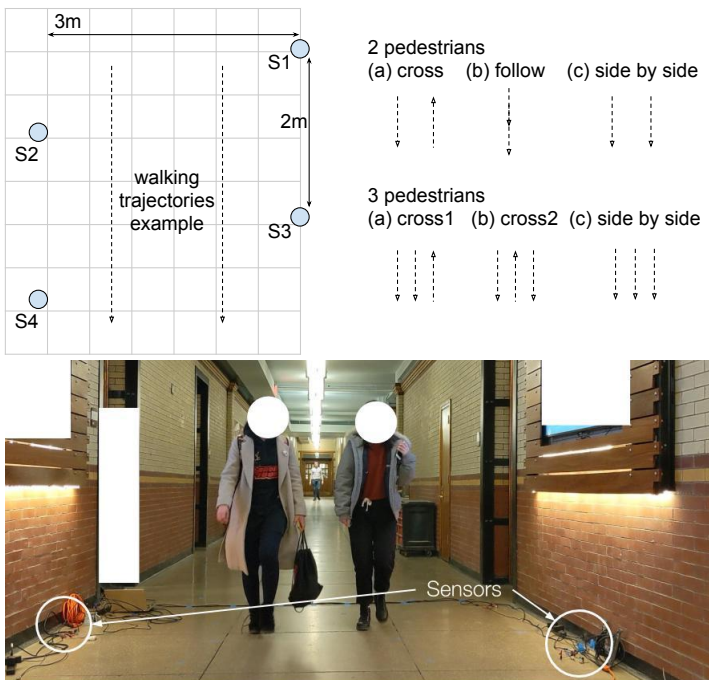

Figure 3: Experimental settings. Top left figure shows the sensor placement. Top right figure shows the walking scenarios investigated when multiple people walk through the sensing area simultaneously. The bottom figure shows a photo taken during the experiments.

Each scenario is evaluated with five repetitions. We investigate different walking direction combinations (Figure 3), including:

- Cross: people walk towards each other from two sides of the area at the same time

- Side by Side: people walk through the area side by side

- Follow: one person walks through the sensing area while another follows him/her three steps away.

\subsection{Baseline Methods}

To evaluate how well our algorithm that takes into account the vibration physical properties, we compare our localization algorithm to two baseline methods as follows:

- NoFilter: applying the MDS on the TDoA estimated from the raw signal without adapting the scales;

- NoAdaptive: using the MDS on TDoAs estimated from a randomly selected wavelet scale signal.

\section{RESULTS AND ANALYSIS}

The goals of the experiments include 1) understanding system performance under different signal overlapping conditions, and 2) evaluating system's ability to handle different number of occupants and different walking scenarios. 

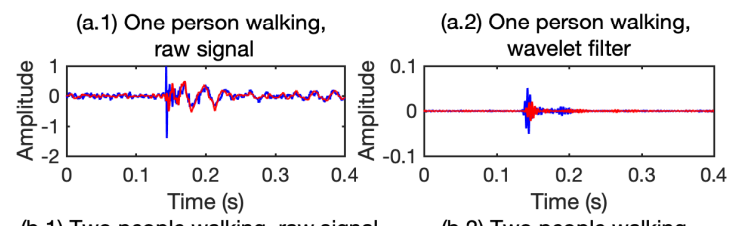

(b.1) Two people walking, raw signa
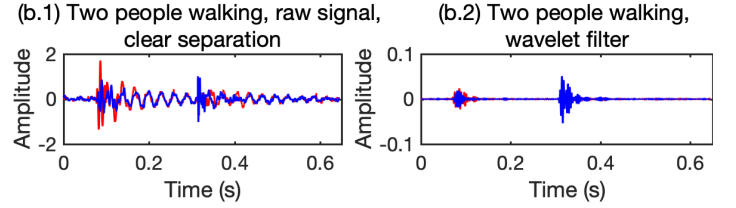

(c.1) Two people walking, raw signal,

(c.2) Two people walking,
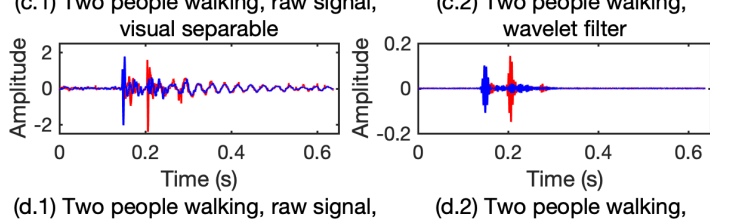

(d.1) Two people walking, raw signal,
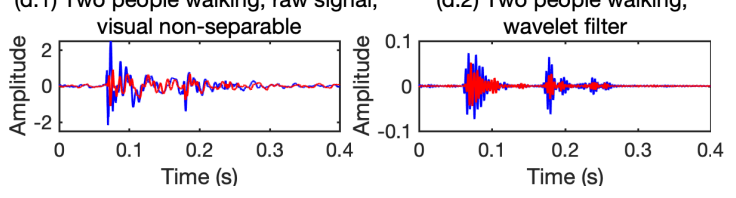

Figure 4: Example signals detected by two sensors when overlap conditions vary.

\subsection{Overlapping Status Analysis}

We evaluate the system in three categories of signal overlap. Figure 4 demonstrates examples of these possible signal overlaps: 1) clear separation, where the offset between signals is no less than $1 / 4$ of the interval of two consecutive footsteps from the same person (e.g., Figure 4 (b.1)); 2) visually separable, where we can visually separate the signal onsets, but the offset is less than $1 / 4$ interval of two consecutive footsteps from the same person (e.g., Figure 4 (c.1)); 3) visually non-separable (e.g., Figure 4 (d.1)).

In figure 4 we observe that the filtered signal is clearer and contains less noise than the unfiltered signal, making footstep detection in overlapping signals easier. Figure 4(x.1) shows the raw footstepinduced vibration signals when people walk by the sensing area, captured by two sensors. The corresponding filtered signals are shown in Figure 4(x.2).

\subsection{System Characterization}

Figure 5 shows the distribution ${ }^{1}$ of the localization error when two people walk through the sensing area with varying levels of footstep overlap. When multiple pedestrians are walking in the target sensing area, we calculate the localization rate and localization error to evaluate our system. For all the investigated walking scenarios with different numbers of pedestrians, Figure 6 shows the localization rate (6(a)) and localization error (6(b)). Our system achieved $100 \%$ localization rate for all the cases, which demonstrates the localization robustness. Our system achieves an average localization error of just $0.65 \mathrm{~m}$, which represents $2.9 \times$ and $2.4 \times$

\footnotetext{
${ }^{1}$ Note that in this paper, the boxplot presents the mean as the circle marker and the median as the center line, the box upper and lower edges indicate the 75 percentile and 25 percentile respectively.
}

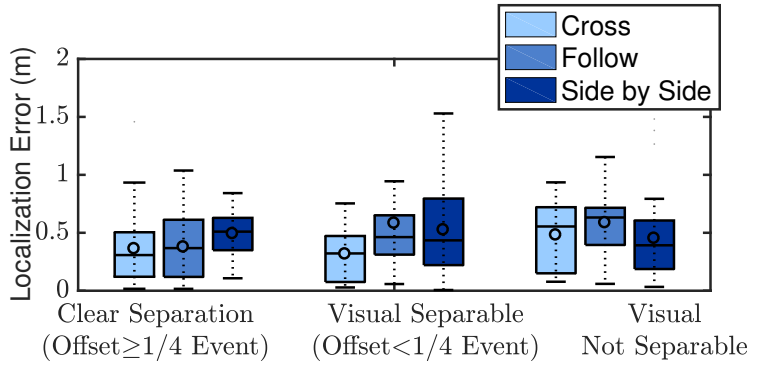

Figure 5: Footstep localization error when two people walk in the sensing area in different scenarios.

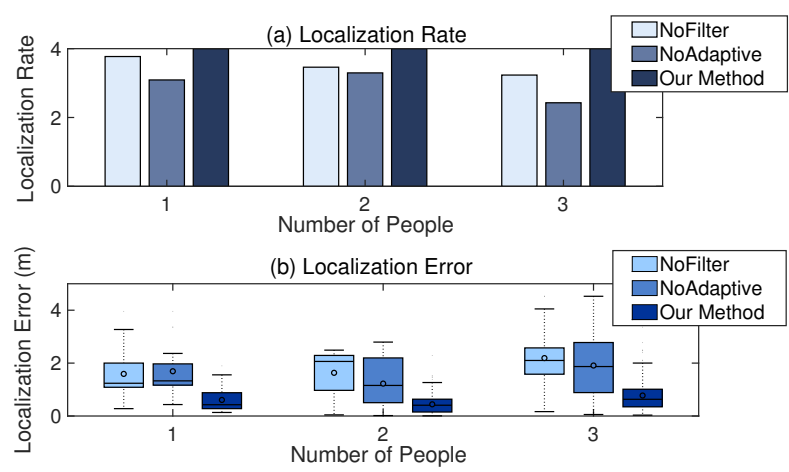

Figure 6: Footstep localization error when different numbers of people walk in the sensing area at the same time.

improvement over baseline approaches NoFilter $(1.91 \mathrm{~m})$ and NoAdaptive (1.56m). From these results, we conclude that our approach accurately localizes multiple concurrent walkers with submeter accuracy, which enables robust indoor occupant monitoring in a variety of real-world walking scenarios.

\section{CONCLUSION}

In this paper, we present a device-free system that localizes multiple occupants through footstep-induced structural vibrations. Our system utilizes physical knowledge on structural frequency excitation response to select non-fundamental frequency bands that have high SNR and high damping rate for heel strikes induced vibration signal. The signal filtering on these selected frequency band enables separation of footstep onsets from the partially overlapping signals for detection and localization. We evaluate our system and achieve sub-meter level localization accuracy through a series of real-world experiments, which enables various indoor application scenarios.

\section{ACKNOWLDGEMENTS}

This work is supported by National Science Foundation (NSF) under the grants CMMI-1653550 and ECCS-1818571, Highmark, Intel, and Google. The views and conclusions contained here are those of the authors and should not be interpreted as necessarily representing the official policies or endorsements, either express or implied, of CMU, UCM, NSF, or the U.S. Government or any of its agencies. 


\section{REFERENCES}

[1] Jerome Berclaz, Francois Fleuret, and Pascal Fua. 2009. Multiple object tracking using flow linear programming. In Performance Evaluation of Tracking and Surveillance (PETS-Winter), 2009 Twelfth IEEE International Workshop on. IEEE, 1-8.

[2] Maurizio Bocca, Ossi Kaltiokallio, Neal Patwari, and Suresh Venkatasubramanian. 2014. Multiple target tracking with RF sensor networks. IEEE Transactions on Mobile Computing 13, 8 (2014), 1787-1800.

[3] Robert Bodor, Bennett Jackson, and Nikolaos Papanikolopoulos. 2003. Visionbased human tracking and activity recognition. In Proc. of the 11th Mediterranean Conf. on Control and Automation, Vol. 1. Citeseer.

[4] Rune Brincker, Lingmi Zhang, and P Andersen. 2000. Modal identification from ambient responses using frequency domain decomposition. In Proc. of

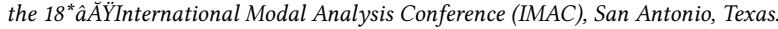

[5] Wenqiang Chen, Maoning Guan, Lu Wang, Rukhsana Ruby, and Kaishun Wu. 2017. FLoc: Device-free passive indoor localization in complex environments. In 2017 IEEE International Conference on Communications (ICC). IEEE, 1-6.

[6] Ivan Dokmanic and M Vetterli. 2015. Listening to distances and hearing shapes: Inverse problems in room acoustics and beyond. EPFL, Lausanne (2015).

[7] James S Hall and Jennifer E Michaels. 2011. Model-based parameter estimation for characterizing wave propagation in a homogeneous medium. Inverse Problems 27,3 (2011), 035002.

[8] Qi Hao, Fei Hu, and Jiang Lu. 2010. Distributed multiple human tracking with wireless binary pyroelectric infrared (PIR) sensor networks. In Sensors, 2010 IEEE. IEEE, 946-950.

[9] Jeffrey M Hausdorff. 2007. Gait dynamics, fractals and falls: finding meaning in the stride-to-stride fluctuations of human walking. Human movement science 26 , 4 (2007), 555-589.

[10] Zhengqiang Jiang, Du Q Huynh, William Moran, Subhash Challa, and Nick Spadaccini. 2010. Multiple pedestrian tracking using colour and motion models. In Digital Image Computing: Techniques and Applications (DICTA), 2010 International Conference on. IEEE, 328-334.

[11] Shoji Makino, Shoko Araki, Ryo Mukai, and Hiroshi Sawada. 2004. Audio source separation based on independent component analysis. In 2004 IEEE International Symposium on Circuits and Systems (IEEE Cat. No. 04CH37512), Vol. 5. IEEE, V-V.

[12] Mostafa Mirshekari, Shijia Pan, Jonathon Fagert, Eve M Schooler, Pei Zhang, and Hae Young Noh. 2018. Occupant localization using footstep-induced structural vibration. Mechanical Systems and Signal Processing 112 (2018), 77-97.

[13] Mostafa Mirshekari, Shijia Pan, Pei Zhang, and Hae Young Noh. 2016. Characterizing wave propagation to improve indoor step-level person localization using floor vibration. In Sensors and Smart Structures Technologies for Civil, Mechanical, and Aerospace Systems 2016, Vol. 9803. International Society for Optics and Photonics, 980305.

[14] Toshiaki Miyazaki and Yuki Kasama. 2015. Multiple human tracking using binary infrared sensors. Sensors 15, 6 (2015), 13459-13476.

[15] Tommy Öberg, Alek Karsznia, and Kurt Öberg. 1993. Basic gait parameters: reference data for normal subjects, 10-79 years of age. Fournal of rehabilitation research and development 30 (1993), 210-210.

[16] Shijia Pan, Kent Lyons, Mostafa Mirshekari, Hae Young Noh, and Pei Zhang. 2016. Multiple pedestrian tracking through ambient structural vibration sensing. In Proceedings of the 14th ACM Conference on Embedded Network Sensor Systems CD-ROM. ACM, 366-367.

[17] Shijia Pan, Mostafa Mirshekari, Pei Zhang, and Hae Young Noh. 2016. Occupant traffic estimation through structural vibration sensing. In SPIE Smart Structures and Materials+ Nondestructive Evaluation and Health Monitoring. International Society for Optics and Photonics, 980306-980306.

[18] Jeffrey D Poston, Javier Schloemann, R Michael Buehrer, VVN Malladi, Americo C Woolard, and Pablo A Tarazaga. 2015. Towards indoor localization of pedestrians via smart building vibration sensing. In Localization and GNSS (ICL-GNSS), 2015 International Conference on. IEEE, 1-6.

[19] Javier Schloemann, VVN Sriram Malladi, Americo G Woolard, Joseph M Hamilton, R Michael Buehrer, and Pablo A Tarazaga. 2015. Vibration Event Localization in an Instrumented Building. In Experimental Techniques, Rotating Machinery, and Acoustics, Volume 8. Springer, 265-271.

[20] Zheng Sun, Aveek Purohit, Kaifei Chen, Shijia Pan, Trevor Pering, and Pei Zhang. 2011. PANDAA: physical arrangement detection of networked devices through ambient-sound awareness. In Proceedings of the 13th international conference on Ubiquitous computing. ACM, 425-434.

[21] Chenren Xu, Bernhard Firner, Robert S Moore, Yanyong Zhang, Wade Trappe, Richard Howard, Feixiong Zhang, and Ning An. 2013. Scpl: Indoor device-free multi-subject counting and localization using radio signal strength. In Information Processing in Sensor Networks (IPSN), 2013 ACM/IEEE International Conference on. IEEE, 79-90.

[22] Candy Yiu and Suresh Singh. 2007. Tracking people in indoor environments. In International Conference on Smart Homes and Health Telematics. Springer, 44-53. 